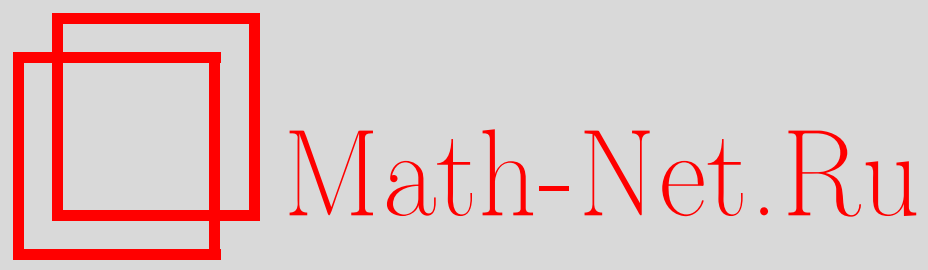

В. И. Арнольд, Топологическая классификация комплексных тригонометрических многочленов и комбинаторика графов с одинаковым числом вершин и ребер, Функи. анализ и его прил., 1996, том 30, выпуск 1, 1-17

DOI: https://doi.org/10.4213/faa501

Использование Общероссийского математического портала MathNet.Ru подразумевает, что вы прочитали и согласны с пользовательским соглашением http://www. mathnet.ru/rus/agreement

Параметры загрузки:

IP : 35.173 .137 .237

26 апреля 2023 г., 18:18:42

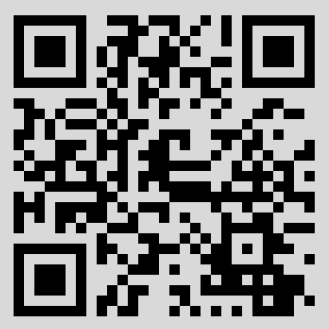




\title{
Топологическая классификация комплексных тригонометрических многочленов и комбинаторика графов с одинаковым числом вершин и ребер
}

\author{
(с) 1996. В. И. Арнольд
}

\section{§1. Введение}

Многочлен - это голоморфное отображение сферы на сферу, для которого одна из точек сферы-образа имеет ровно один прообраз.

Топологическая классификация комплексных многочленов общего положения была сведена в работах Девиса, Тома и Здравковски [1-3] к чисто комбинаторной задаче теории графов.

Эта комбинаторная задача была исследована уже Кэли, перечислившим деревья с $n$ нумерованными ребрами: их число равно $(n+1)^{n-2}$.

Быть может, самым простым доказательством этой теоремы Кэли является подсчет кратности квазиоднородного отображения Ляшко-Лоенги, сопоставляющего многочлену степени $n+1$ неупорядоченный набор $n$ его критических значений $[4-6]$.

В настоящей работе проведено аналогичное исследование топологических свойств комплексных тригонометрических многочленов, что приводит к обобщениям теоремы Кэли, т. е. к новым комбинаторным результатам теории графов.

Тригонометрический многочлен - это голоморфное отображение сферы на сферу, для которого одна из точек сферы-образа имеет ровно два прообраза.

Топологическая классификация таких отображений степени $n$ общего положения оказывается эквивалентной перечислению связных графов с $n$ нумерованными ребрами, соединяющими $n$ вершин (обе вершины каждого ребра графа здесь и ниже предполагаются всегда различными).

Каждое ребро графа задает транспозицию множества вершин, переставляющую конщы этого ребра. Произведение этих транспозиций (в порядке, заданном нумерацией ребер) называется перестановкой Коксетера графа (потому что Коксетер эту перестановку не рассматривал).

Перестановка Коксетера связного графа с одинаковым числом $n$ вершин и ребер всегда состоит ровно из двух циклов длин $p$ и $q, p+q=n$. Числа $p$ и $q$ - это порядки полюсов соответствующего графу лорановского многочлена. (Для обычных многочленов степени $n+1$, или для деревьев с $n$ нумерованными ребрами, перестановка Коксетера состоит из одного цикла - факт, хорошо известный в теории алгебр Ли.)

Число связных графов с нумерованными ребрами и перестановкой Коксетера из двух циклов длин $p$ и $q$ оказывается равным $N(p, q) /(p q)$, если $p \neq q$, и $N(p, q) /(2 p q)$, если $p=q>1$. 
Здесь $N$ - степень соответствующего отображения Л3 Лорана-Ляшко-Лоенги (этими авторами не рассматривавшегося и определенного ниже в $\S 3$ ):

$$
N(p, q)=p^{p} q^{q}(p+q-1) ! /(p-1) !(q-1) !
$$

Например, $N(p, 1)=p^{p+1}, N(p, 2)=4(p+1) p^{p+1}, N(2,1)=8, N(2,2)=96$. Это же в сущности отображение с другой точки зрения ранее исследовалось Горюновым $[7,8]$, но он не связывал предмет с перечислением графов.

Из наших построений вытекает, в частности, что пространство комплексных тригонометрических многочленов общего положения (с фиксированными порядками полюсов $p$ и $q$ ) является пространством Эйленберга-Маклейна $K(\pi, 1)$ для некоторой явно описанной подгруппы конечного индекса группы кос из $n=p+q$ нитей.

Это позволяет вычислять когомологии указанных подгрупп (что может привести к характеристическим классам алгебраических функций, аналогичным построенным в [9] для целых алгебраических функций характеристическим классам, а следовательно, к теоремам о непредставимости алгебраических функций полными суперпозициями алгебраических не обязательно целых функций).

Проведенный ниже анализ, по-видимому, можно распространить на рациональные функции с бо́льшим двух числом полюсов. Для трех полюсов (что соответствует графам с двумя циклами, у которых число вершин на 1 меньше, чем число ребер) теория должна быть еще очень похожей на построенную ниже.

Однако уже в этом случае возникает существенно новое обстоятельство: не всякая нумерация ребер графа соответствует рациональной функции (и перестановке Коксетера с тремя циклами).

Граф с нумерованными ребрами имеет интересный инвариант - род $g$ той римановой поверхности, которую определяет разветвленное накрытие сферы, построенное по этому графу.

Формула Римана-Гурвица (т. е. аддитивность эйлеровой характеристики) дает следующее выражение для рода $g$ связного графа с $m$ вершинами и $n$ нумерованными ребрами:

$$
2-2 g=m-n+k,
$$

где $k$ - число циклов перестановки Коксетера (равное числу полюсов соответствующей графу мероморфной функции и разности эйлеровых характеристик римановой поверхности и графа).

В частности, четность числа циклов перестановки Коксетера противоположна четности числа независимых циклов графа (и не зависит от нумерации). Из неравенства $k>0$ следует оценка рода сверху, $2 g<n-m+2$.

Например, при $m \geqslant n$ (т.е. для деревьев и для графов с одним циклом) род равен нулю. Поэтому $k=1$ при $m=n+1$ и $k=2$ при $m=n$. Таким образом, топологическая формула Римана-Гурвица доставляет доказательство следующего комбинаторного факта теории графов: перестановка Коксетера связного графа с нумерованными ребрами имеет ровно один цикл, если граф является деревом, и ровно два, если граф имеет один цикл.

При бо́льшем числе ребер род может быть и положительным. Например, для связных графов с двумя независимыми циклами $n=m+1$ и $g \leqslant 1$. Поэтому перестановка Коксетера связного графа с двумя независимыми циклами состоит либо из $k=3$ циклов (и тогда $g=0$ ), либо из $k=1$ циклов (и тогда $g=1$ ). 
Первый случай соответствует рациональным функциям с тремя полюсами, а второй - эллиптическим с одним полюсом.

Например, существуют ровно три связных графа с тремя вершинами и четырьмя ребрами:
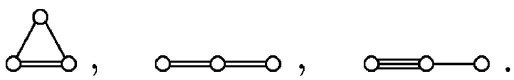

Эти графы имеют $6+3+4=13$ нумераций. Из них четыре имеют род 0 $(k=3)$, а девять - род $1(k=1)$. При $m=4, n=5$ числа нумерованных связных графов родов 0 и 1 равны 120 и 160 соответственно. (Чтобы получить эти числа, я просто нарисовал все эти 280 графов и нумераций ребер, общей формулы я не знаю.)

При бо́льшем трех числе полюсов их конфигурация имеет модули, и положение полюсов нельзя фиксировать. Результаты о свойстве Эйленберга-Маклейна в этом случае, вероятно, можно, если не фиксировать полюсы, получить методом работы Натанзона [10]. Однако вопросы о свойствах отображений типа ЛяшкоЛоенги и о функциях с фиксированными полюсами в этих случаях нуждаются в дальнейшем исследовании.

Результаты настоящей работы были получены при попытке неформальной комплексификации недавних исследований Казаряна [11-13] семейств функщий на окружности. Вероятно, из полученных результатов комплексной геометрии можно извлечь и сведения о пространствах вещественных тригонометрических многочленов.

Вещественный вариант обычной теории Ляшко-Лоенги, описанный в [14], сводит изучение топологии максимальных морсификаций критических точек функций одной переменной к геометрии конусов Спрингера групп Вейля $A_{n}$.

Гипотетическое обобщение этих построений на тригонометрический случай должно доставить, в частности, перечисление компонент связности пространства вещественных тригонометрических многочленов степени $n$ с $n$ различными вешественными критическими значениями. Числа компонент в пространстве всех гладких функций на окружности (включая также и мёбиусовские функции, т. е. сечения нетривиального расслоения) при числах критических значений $n=1,2,3,4, \ldots$ образуют последовательность чисел Эйлера-Бернулли [1416] $1,1,1,2,5,16,61, \ldots$. Гипотетически такими же должны быть и числа компонент в пространствах тригонометрических многочленов фиксированной степени (включая мёбиусовские тригонометрические многочлены при нечетных $n$, ср. [17]). Вещественный вариант отображения Ляшко-Лоенги, построенный в [14], гомеоморфно отображает стратификацию множества вещественных многочленов степени $n+1$, не имеющих комплексных критических точек, на разбиение главного конуса Спрингера группы Коксетера $A_{n}$ зеркалами на камеры Вейля (причем над внутренностью конуса Спрингера это отображение даже диффеоморфно).

Гипотетическое обобщение этого построения на тригонометрический случай должно доставить описание стратификащии пространства тригонометрических многочленов степени $n$, не имеющих комплексных критических точек, при помощи зеркал аффинной группы Вейля.

Упорядочивая критические значения по их величине, мы получаем упорядо- 
ченную последовательность критических точек $x_{i} \bmod 2 \pi$ на окружности. Как совпадения критических точек, так и совпадения критических значений, происходят при $x_{i}=x_{j} \bmod 2 \pi$, т. е. на зеркалах аффинной группы.

Вещественный аналог изложенной ниже теории должен содержать утверждение о гомеоморфности отображения пространства вещественных тригонометрических многочленов степени $n$, не имеющих комплексных критических точек, в пространство, где действует аффинная группа Вейля. При этом на многообразии вещественных тригонометрических многочленов с $n$ различными вещественными критическими точками должен получаться даже диффеоморфизм стратификаций.

Автор благодарен А. Г. Хованскому, Б. З. Шапиро, А. Б. Вайнштейну и С. М. Натанзону за полезные обсуждения.

\section{§2. Нумерованный граф мероморфной функции}

Мероморфной функцией называется голоморфное отображение $f: M^{2} \rightarrow S^{2}$ компактной связной римановой поверхности на сферу Римана. Прообразы выделенной на сфере точки $\infty$ называются полюсами функции. Рассмотрим мероморфные функщии с фиксированным числом $k$ полюсов и с фиксированными порядками полюсов.

Почти все такие функции имеют лишь невырожденные конечные (отличные от полюсов) критические точки (где $f^{\prime}=0, f^{\prime \prime} \neq 0$ ) и попарно не совпадающие критические значения. Мы будем обозначать это общее число конечных критических значений через $n$, а суммарную кратность отображения $f$ (его степень, т. е. число прообразов точки общего положения, равное также сумме порядков полюсов) будем обозначать через $m$.

Из формулы Римана-Гурвица следует, что род $g$ поверхности $M$ связан с числами $m, n$ и $k$ соотношением

$$
2-2 g=m-n+k .
$$

Характер ветвления мероморфной функции удобно описывать при помощи графа с $n$ нумерованными ребрами и $m$ вершинами, который мы сейчас опишем.

Зафиксируем на сфере точку $O$ общего положения (т.е. не являющуюся ни образом полюсов, ни критическим значением нашего отображения). Она имеет $m$ прообразов $\left\{z_{i}\right\}$ на поверхности $M$. Эти прообразы и будут вершинами нашего графа.

Занумеруем все $n$ конечных критических значений отображения $f$ какимлибо образом в последовательность $\left(F_{1}, \ldots, F_{n}\right)$. Проведем через эти $n$ точек сферы гладко вложенную окружность, проходящую их в этом порядке и так, что точка $O$ оказывается внутри, а точка $\infty$ - снаружи диска, границей которого является эта ориентированная окружность (диск ориентирован комплексной структурой сферы Римана). Этот набор ( $O$, нумерация $F_{i}$, диск) назовем поляризачией нашей функции.

Дополнительная (содержащая $\infty$ ) область также является диском. Мы можем диффеоморфно отобразить каждый из этих двух дисков на стандартный диск так, что точка $O$ (соответственно $\infty$ ) перейдет в центр. Будем называть «ради- 
усами» $O F_{i}$ (соответственно $\left.\infty F_{i}\right)$ наших двух дисков прообразы настоящих радиусов стандартного диска при этих диффеоморфизмах (рис. 1). Эти «радиусы»- некоторые гладкие пути, соединяющие $O$ (соответственно $\infty$ ) с критическими значениями и не имеющие общих точек (кроме, конечно, общего начала в $O$ или в $\infty$ и общего конца «радиусов» $O F_{i}$ и $\left.\propto F_{i}\right)$.

При движении точки $O$ вдоль «радиуса» $O F_{i}$ ее прообразы $\left(z_{1}, \ldots, z_{m}\right)$ непрерывно движутся, оставаясь попарно различными, пока два из них не сольются над точкой $F_{i}$ в критической точке $C_{i}$ отображения $f \mathrm{c}$ критическим значением $F_{i}$. По определению $i$-е ребро нашего графа соединяет те два прообраза $\left(z_{u(i)}, z_{v(i)}\right)$ точки $O$, которые при движении $O$ вдоль «радиуса» $O F_{i}$ приходят в одну критическую точку $C_{i}$ отображения $f$.

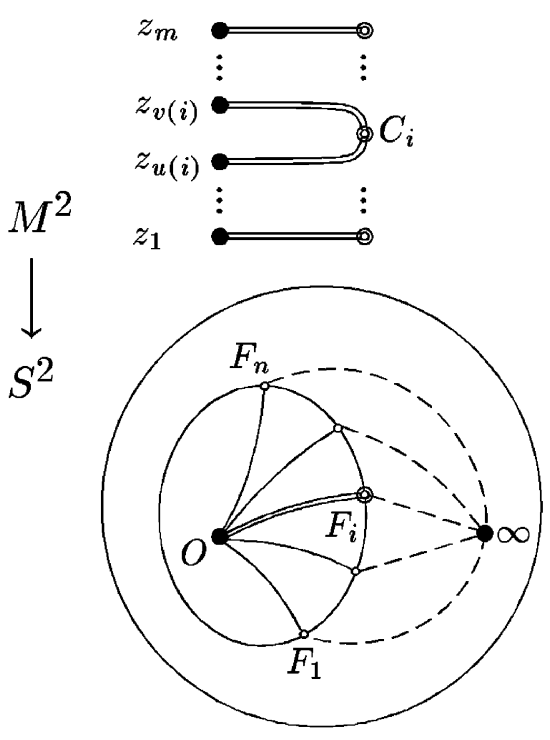

Рис. 1

ОПРЕДЕЛЕНИЕ. Нумерованнылм графом мероморфной функции $f$ называется граф с $m$ вершинами $\left\{z_{1}, \ldots, z_{m}\right\}$ и $n$ нумерованными ребрами $\left(z_{u(i)}, z_{v(i)}\right)$.

ЗАМЕЧАНИЕ 1. ЭТот граф зависит от выбора класса поляризации: точки $O$ и окружности, проходящей через критические значения, а также и от выбора на ней начальной точки. На множестве классов таких поляризаций и на множестве графов естественно действует группа кос из $n$ нитей. Орбита этого действия группы кос является уже топологическим инвариантом самой функции, рассматриваемой с точностью до гомеоморфизмов и прообраза, и образа. Но мы будем ниже изучать функции с фиксированной поляризацией, классифицируя их с точностью до гомеоморфизмов одного лишь прообраза, $M$.

ЗАмЕчание 2. Наш граф естественно вложен в поверхность-прообраз $M$. Ребро с номером $i$ во вложенном графе состоит из тех двух прообразов «радиуса» $O F_{i}$, которые соединяются в критической точке $C_{i}$ с критическим значением $F_{i}$.

Например, если $f: S^{2} \rightarrow S^{2}$ - многочлен степени $n+1$, то $m=n+1$ и граф - дерево с нумерованными ребрами, вложенное в сферу-прообраз и соединяющее все $n$ конечных критических точек этого многочлена.

ЗАмЕчАнИЕ 3. Граф мероморфной функции связен. Это следует из связности поверхности $M$. Действительно, разрежем сферу-образ по «радиусам» $\infty F_{i}$. Дополнение к объединению разрезов - топологический диск. Его прообраз состоит из $m$ дисков, называемых листами римановой поверхности. Вершины нашего графа можно истолковывать, таким образом, как эти листы.

Ребра графа описывают склейки берегов разрезов листов. Если бы граф был несвязен, то соответствующие листы римановой поверхности не соединялись бы и она распалась бы на связные компоненты, соответствующие связным компонентам графа. 
ОПРЕДЕЛЕНИЕ. $(m, n)$-графом называется связный граф с $m$ вершинами и $n$ ребрами, в котором оба конца каждого ребра различны. Нумерачией $(m, n)$-графа называется нумерация его ребер числами $(1, \ldots, n)$.

ОПРЕДЕЛЕНИЕ. Перестановкой Коксетера нумерованного $(m, n)$-графа называется перестановка его вершин, являющаяся произведением транспозиций, переставляющих концы ребер, в порядке, заданном нумерацией ребер:

$$
\sigma=\sigma_{n} \circ \cdots \circ \sigma_{1}, \quad \sigma_{i}\left(z_{u(i)}\right)=z_{v(i)}, \sigma_{i}\left(z_{v(i)}\right)=z_{u(i)} \cdot
$$

ОПРЕДЕЛЕНИЕ. Порядком нумерованного $(m, n)$-графа называется набор длин циклов его перестановки Коксетера (это — разбиение числа $m$ его вершин на слагаемые, т. е. диаграмма Юнга площади $m)$.

ОПрЕДЕЛЕНИЕ. Типом нумерованного $(m, n)$-графа называется число $k$ циклов его перестановки Коксетера.

ОПРедЕлениЕ. Родом нумерованного $(m, n)$-графа называется число $g$, определяемое через число вершин $m$, число ребер $n$ и тип $k$ по формуле (1).

Очевидна

ТЕОРемА 1. Порядок нумерованного $(m, n)$-графа мероморфной функиии есть набор порядков полюсов этой функиии, тип - число полюсов, а род - род римановой поверхности $M$.

Несколько менее очевидна обратная, вытекающая из теории Римана,

ТЕОремА 2. Всякий нумерованный $(m, n)$-граф является нумерованньм $(m, n)$-графом мероморфной бункиии на поверхности рода $g$ c $k$ полюсами, порядки которых составляют порядок графа.

ДОПОЛНЕНИЕ К ТЕОРЕМЕ 2. Всякий нумерованный $(m, n)$-араф реализуется как нумерованный $(m, n)$-граф некоторой мероморфной функции, поляризачия которой является яюбой наперед заданной системой, состоящей из не содержашего $\infty$ диска и точек $\left(F_{1}, \ldots, F_{n}\right)$, расположенных в этом порядке на его крае.

ДокАЗАтельство. Разрежем сферу-образ по «радиусам» $\infty F_{i}$ диска, содержащего точку $\infty$. Возьмем $m$ копий разрезанной сферы соответственно $m$ вершинам графа. Склеим эти копии по правилу, определяемому графом: для $i$-го ребра, соединяющего вершины $u(i)$ и $v(i)$, склеиваются накрест берега разрезов над "радиусом» $\infty F_{i}$ на листах $u(i)$ и $v(i)$ (а на каждом из остальных листов склеиваются оба берега разреза над этим «радиусом»). Получается замкнутая риманова поверхность $M$.

Комплексная структура на полученной поверхности индуцируется комплексной структурой сферы-образа (в точки ветвления она продолжается по общим теоремам теории функций комплексного переменного).

Проекция построенной поверхности $M$ на сферу имеет заданный нумерованный $(m, n)$-граф и заданную наперед поляризацию. Дополнение (а с ним и теорема) доказано.

ЗАмЕчАНИЕ. Из этих топологических и аналитических рассуждений вытекают интересные комбинаторные свойства нумерованных $(m, n)$-графов. Например, из формулы (1) получаем

СЛЕДСТВИЕ 1. Тип $k$ нумерованного $(m, n)$-графа удовлетворяет неравенству $k \leqslant 2-m+n$ (m. е. не превосходит иисла независимых ииклов графа 
плюс единииа).

В частности, для деревьев $m=n+1$ и потому $k=1, g=0$.

СЛЕДСТВИЕ 2. Тип всякого нумерованного $(n, n)$-графа равен двум, а род - нулю.

Действительно, $k$ не превосходит 2 и четно; поэтому $k=2$. Согласно равенству (1), $g=0$.

Именно этот частный случай доставляет топологическую классификацию тригонометрических многочленов.

ОПредЕлениЕ. Две мероморфные функции $f_{1}: M \rightarrow S^{2}, f_{2}: M \rightarrow S^{2}$ называются топологически эквивалентныли, если одна из них переводится в другую гомеоморфизмом $h: M \rightarrow M$ поверхности-прообраза (так что $f_{2}=f_{1} \circ h$ ).

ЗАмечАниЕ. Топологически эквивалентные мероморфные функции имеют одинаковые критические значения. Поэтому для них можно выбрать на сфереобразе общую поляризацию. Поляризация и граф с нумерованными ребрами определяют мероморфную функцию с точностью до топологической эквивалентности.

Суммируя сказанное, мы получаем такой вывод:

ТЕОРемА. Число попарно топологически не эквивалентных мероморфных функиий с данными порядками полюсов $p_{i}$ и $е$ п данными попарно различными конечными критическими значениями равно числу попарно не изоморфных нумерованных $(m, n)$-графов порядка $\left\{p_{i}\right\}$ (где $\left.m=\sum p_{i}\right)$.

В случае рациональных функций $g=0$. Число полюсов тригонометрического (лорановского) многочлена есть $k=2$. По формуле (1) $m=n$, так что получается

ТЕОРема. Число попарно топологически не эквивалентных рачиональных функчий с двумя полюсами порядков $p$ и $q$ с $n=p+q$ конечньми фиксированныли попарно различными критическими значениями равно числу попарно не изоморфных нумерованных $(n, n)$-графов, перестановки Коксетера которых состоят из двух ииклов порядков р и $q$.

ПримеР. Число нумерованных $(3,3)$-графов с $p=2, q=1$ равно 4. Число $(4,4)$-графов с $p=2, q=2$ равно 12 , а с $p=3, q=1$ равно 27.

Полюсы наших функций можно поместить в точки 0 и $\infty$ сферы-прообраза, $M$, при помощи ее дробно-линейного диффеоморфизма, переводящего каждую функцию в топологически ей эквивалентную. Поэтому фактически речь идет о топологической классификации многочленов Лорана с полюсами фиксированных порядков в точках 0 и $\infty$. Именно топологическое исследование этих многочленов Лорана позволяет решить комбинаторную задачу о числе графов, к которой мы пришли. Ответ указан во введении.

\section{§3. Отображение Лорана-Ляшко-Лоенги Л3}

Многочлены Лорана удобнее записывать как функции на гиперболе.

ОПРЕДЕЛЕНИЕ. Бимногочленом бистепени $(p, q)$ называется ограничение многочлена от двух переменных

$$
H(Z, W)=W^{p}+a_{1} W^{p-1}+\cdots+a_{p-1} W+c+b_{q-1} Z+\cdots+b_{1} Z^{q-1}+Z^{q}
$$

на «гиперболу» $W Z=\gamma$. Мы будем всегда считать, что $p$ и $q$ положительны. 
Все бимногочлены бистепени $(p, q)$ образуют пространство $\mathbb{C}^{p+q=n}$ с координатами $(a, b, c ; \gamma)$. Если $\gamma \neq 0$, то бимногочлен называется собственнылм, а если $\gamma=0$, то несобственнылм.

Собственный бимногочлен имеет на гиперболе ровно $n=p+q$ критических точек (считая с кратностями). Пусть $\left\{x_{1}, \ldots, x_{n}\right\}$ - множество соответствующих критических значений.

ОпреДЕЛЕниЕ. Отображением Л3 (Лорана-Ляшко-Лоенги) $\mathbb{C}^{n} \rightarrow \mathbb{C}^{n}$ называется отображение, сопоставляющее бимногочлену многочлен

$$
f(x)=x^{n}+u_{1} x^{n-1}+\cdots+u_{n},
$$

корнями которого являются критические значения этого бимногочлена.

Строго говоря, определение пока годится только для собственных бимногочленов. Однако имеет место

Теорема. Отображение Л3 является полиномиальным, т.е. задается $p+q=n$ многочленами $u_{i}(a, b, c ; \gamma)$ при $\gamma \neq 0$ (а следовательно, продолжается по непрерывности на все пространство $\left.\mathbb{C}^{n}\right)$.

ПримеР. При $p=q=1$ имеем $u_{1}=-2 c, u_{2}=c^{2}-4 \gamma$.

ЗАмЕЧАнИЕ. Часто удобнее укороченное отображение Л3: $\mathbb{C}^{n-1} \rightarrow \mathbb{C}^{n-1}$ из пространства бимногочленов со свободным членом $c=0$ в пространство многочленов со следом $u_{1}=0$ (чтобы получить многочлен со следом нуль, в качестве корней берутся критические значения $H_{i}$ бимногочлена, сдвинутые так, чтобы их центр тяжести попал в нуль: $\left.x_{i}=H_{i}-\left(\sum H_{i}\right) / n\right)$.

Из теоремы следует, что это отображение тоже полиномиально.

ПримеР 1. При $p=2, q=1$ укороченное отображение Л3: $\mathbb{C}^{2} \rightarrow \mathbb{C}^{2}$ задается формулами

$$
U_{2}=a^{4}+8 a \Gamma, \quad U_{3}=8 \Gamma^{2}+20 a^{3} \Gamma-a^{6},
$$

где $U_{2}=-48 u_{2}, U_{3}=-864 u_{3}, \Gamma=27 \gamma, a=a_{1}$.

Прообраз кривой $\Sigma$ многочленов $f$ с кратными корнями состоит из каустики $\Gamma=a^{3}$ (состоящей из бимногочленов с кратными критическими точками) и прямой несобственных бимногочленов $\Gamma=0$. Якобиан нашего отображения равен $128\left(\Gamma-a^{3}\right)^{2}$. Кратность отображения Л3: $\mathbb{C}^{2} \rightarrow \mathbb{C}^{2}$ равна

$$
N(2,1)=8=3 \cdot 2+1 \cdot 2 .
$$

Каустика накрывает $\Sigma$ двукратно, с трансверсальной кратностью 3 . Прямая $\Gamma=0$ накрывает $\Sigma$ тоже двукратно, но с трансверсальной кратностью 1 .

ЗАМЕЧАНИЕ 1. Кратность укороченного отображения ЛЗ гиперплоскости $\gamma=0$ несобственных бимногочленов бистепени $(p, q)$ на $(p+q-2)$-мерный ласточкин хвост в $\mathbb{C}^{p+q-1}$ дается формулой

$$
m_{0}(p, q)=\frac{p^{p} q^{q}(p+q-2) !}{p ! q !}=\frac{N(p, q)}{p q(p+q-1)}
$$

(которую я тут не доказываю). Например, $m_{0}=p^{p-1}$ при $q=1$ и $m_{0}=2 p^{p}$ при $q=2$.

ЗАмЕЧАНИЕ 2. Кратность отображения Л3 каустики на многомерный ласточкин хвост при произвольных $(p, q)$ (и даже для $q=1$ ) мне неизвестна. 
Для обычного отображения Ляшко-Лоенги Л2 пространства многочленов с $n$ критическими значениями она равна $(n+1)^{n-2}$. Поскольку кратность этого отображения во всем пространстве равна $(n+1)^{n-1}$, кратность отображения Л2 страта Максвелла на ласточкин хвост равна $(n+1)^{n-2}(n-2) / 2$.

ПримеР 2. Тригонометрические многочлены степени 2 записываются в виде бимногочленов бистепени $(2,2)$ :

$$
H=W^{2}+a W+b Z+Z^{2}, \quad W Z=\gamma .
$$

Укороченное отображение $Л 3: \mathbb{C}^{3} \rightarrow \mathbb{C}^{3},(a, b ; \gamma) \mapsto\left(u_{2}, u_{3}, u_{4}\right)$ задается формулами

$u_{2}=Q^{2}-20 \gamma Q-8 \gamma^{2}-\frac{3}{8} P^{2}, \quad-u_{3}=\frac{1}{2} P Q^{2}+8 \gamma P Q+32 \gamma^{2} P-\frac{1}{8} P^{3}$, $u_{4}=16 \gamma^{4}-48 \gamma^{3} Q+48 \gamma^{2} Q^{2}-\frac{37}{2} \gamma^{2} P^{2}+\frac{13}{4} \gamma P^{2} Q-16 \gamma Q^{3}+\frac{1}{16} P^{2} Q^{2}-\frac{3}{256} P^{4}$, где $4 P=a^{2}+b^{2}, 4 Q=a b$.

Это отображение квазиоднородно, с весами

$$
\begin{gathered}
\operatorname{deg} a=\operatorname{deg} b=\operatorname{deg} W=\operatorname{deg} Z=1, \\
\operatorname{deg} P=\operatorname{deg} Q=\operatorname{deg} \gamma=\operatorname{deg} H=2, \quad \operatorname{deg} u_{i}=2 i .
\end{gathered}
$$

Прообраз ласточкиного хвоста $\Sigma \subset \mathbb{C}^{3}$ (состоящего из многочленов $f=$ $x^{4}+u_{2} x^{2}+u_{3} x+u_{4}$, имеющих кратный корень) состоит из трех частей:

(i) каустика (многообразие бимногочленов, имеющих кратные критические точки) пересекает плоскость $\gamma=1$ по астроиде (гипоциклоиде с четырьмя остриями), $27 P^{2} \gamma=4\left(Q+8 \gamma^{2}\right)^{2}(Q-\gamma)$;

(ii) cmpam Максвелла (многообразие бимногочленов, имеющих кратные критические значения) пересекает плоскость $\gamma=1$ по паре прямых - касательных к астроиде в парах противоположных точек возврата, $P= \pm 2 Q$;

(iii) плоскость $\gamma=0$ несобственных бимногочленов тоже отображается на ласточкин хвост $\Sigma$ (хотя якобиан отображения ЛЗ в ее общих точках и не равен 0).

Кратность укороченного отображения $Л 3: \mathbb{C}^{3} \rightarrow \mathbb{C}^{3}$ во всем пространстве равна

$$
N(2,2)=96=3 \cdot 24+2 \cdot 8+1 \cdot 8
$$

соответственно вкладам трех стратов (i), (ii) и (iii). Кратности отображения каустики, страта Максвелла и плоскости несобственных бимногочленов на ласточкин хвост равны $(24,8,8)$. Трансверсальные кратности на этих стратах всегда равны $(3,2,1)$, т. е. порядки нуля якобиана отображения Л3 (или укороченного отображения Л3) в их типичных точках равны $(2,1,0)$ при любых $p$ и $q$. В случае каустики и страта Максвелла это верно и для отображения Л2.

ДокАЗАТЕЛЬСтво ТЕОРЕМЫ. Критические точки определяются из условия $Z H_{Z}-W H_{W}=0$. Подставляя значение $W=\gamma / Z$, мы приводим это уравнение к виду $A(Z)=0$, где $A$ - многочлен степени $n$ со старшим членом $q Z^{n}$, коэффициенты которого - многочлены от $(a, b, c ; \gamma)$. Обозначим его корни через $Z_{i}$. По теореме о симметрических функциях степенные суммы

$$
\sum_{i=1}^{n} Z_{i}^{k}=P_{k} \quad(k \geqslant 0)
$$


тоже являются многочленами от $(a, b, c ; \gamma)$. Аналогичным образом, заменяя $Z$ на $\gamma / W$, мы заключаем, что и степенные суммы

$$
\sum_{i=1}^{n} W_{i}^{k}=Q_{k} \quad(k \geqslant 0)
$$

являются многочленами от $(a, b, c ; \gamma)$.

Рассмотрим теперь критические значения $H_{i}=H\left(Z_{i}, W_{i}\right)$. Одночлены, входящие в выражение для $H^{k}$, имеют вид $Z^{r} W^{s}$, где $r \geqslant 0, s \geqslant 0$. Такой одночлен равен $\gamma^{s} Z^{r-s}$, если $r \geqslant s$, и $\gamma^{r} W^{s-r}$, если $s \geqslant r$. Коэффициенты при этих одночленах - многочлены от $(a, b, c)$. Окончательно мы представили $H^{k}$ в виде

$$
H^{k}=\sum_{m \geqslant 0}\left(f_{k, m} Z^{m}+g_{k, m} W^{m}\right),
$$

где коэффициенты $f$ и $g$ - многочлены от $(a, b, c ; \gamma)$.

Суммируя по всем $n$ критическим точкам, мы получаем, наконец,

$$
\sum_{i=1}^{n} H_{i}^{k}=\sum_{m \geqslant 0}\left(f_{k, m} P_{m}+g_{k, m} Q_{m}\right) .
$$

Итак, основные симметрические функции от критических значений $H_{i}$ являются многочленами от $(a, b, c ; \gamma)$, что и требовалось доказать.

Выясним смысл продолжения Л3-отображения на гиперплоскость несобственных бимногочленов $\gamma=0$.

Пусть многочлен $A(W)=W^{p}+a_{1} W^{p-1}+\cdots+a_{p-1} W$ имеет в точке $W=0$ нуль порядка $r$, а многочлен $B(Z)=Z^{q}+b_{1} Z^{q-1}+\cdots+b_{q-1} Z-$ нуль порядка $s$ в точке $Z=0$, так что

$$
H(Z, W)=W^{p}+a_{1} W^{p-1}+\cdots+a_{p-r} W^{r}+c+b_{q-s} Z^{s}+\cdots+b_{1} Z^{q-1}+Z^{q},
$$

где $1 \leqslant r \leqslant p, 1 \leqslant s \leqslant q, a_{0}=b_{0}=1, a_{p-r} b_{q-s} \neq 0$.

Лемма. При стремлении $\gamma \kappa 0$ критические точки бимногочлена $H$ на гиперболе $Z W=\gamma$ имеют конечнье предель, а именно:

1) $p-r$ из них стремятся к ненулевым критическим точкам многочлена A на оси $W$;

2) $q-s$ из них стремятся $\kappa$ ненулевым критическим точкам многочлена В на оси $Z$;

3) $r+s$ из них стремятся $\kappa$ нулю.

ДокаЗАтельство. Рассмотрим пересечение кривой $Z H_{Z}-W H_{W}=0$ критических точек на плоскости $(Z, W)$ с координатным крестом $Z W=0$. При $Z=0$ мы получаем для точек пересечения уравнение $W A_{W}=0$. Оно имеет $p-r$ ненулевых корней, являюшихся пределами критических точек, лежаших на гиперболах $Z W=\gamma$ при $\gamma$, стремящемся к 0 . Точно так же при $W=0$ мы получаем $q-s$ ненулевых корней уравнения $Z B_{Z}=0$, лежащих на оси $Z$ и определяюших отличные от 0 предельные положения критических точек.

Кратность же нулевого решения $W=Z=0$ системы

$$
Z B_{Z}-W A_{W}=0, \quad Z W=0
$$


вычисляется как размерность соответствующего локального кольца. Она определяется младшими членами тейлоровских разложений в нуле. Функция $f=$ $Z B_{Z}-W A_{W}$ является полуквазиоднородной степени $r s$ при весах переменных $\operatorname{deg} Z=r, \operatorname{deg} W=s$. По общей формуле для кратности $\mu$ полуквазиоднородного отображения (см., например, [6]) находим

$$
\mu=\frac{r s \operatorname{deg}(Z W)}{\operatorname{deg} Z \operatorname{deg} W}=\frac{r s(r+s)}{r s}=r+s,
$$

что и требовалось доказать.

ОПРЕДЕЛЕНИЕ. Критическими точками (значениями) несобственного бимногочлена называются пределы критических точек (значений) соответствующих собственных бимногочленов при стремлении $\gamma$ к нулю (с учетом кратностей).

ЗАмЕчАНИЕ. Даже в простейшем случае, когда $r=s=1$ (т. е. когда 0 не является критической точкой ни для $A$, ни для $B$ ), несобственный бимногочлен имеет в нуле двукратную критическую точку (с критическим значением $c$ ), поскольку $\mu=r+s=2$.

При таком толковании критических значений бимногочленов отображение Л3 оказывается всегда переводящим бимногочлен в многочлен, корни которого критические значения этого бимногочлена (с их кратностями).

ТЕоремА. Л3-отображение конечнократно в нуле, т. е. прообраз точки $u=0$ cостоит из единственной точки $a=b=c=\gamma=0$.

ДокАЗАТЕЛЬСтво. Все $n$ корней многочлена с $u=0$ нулевые; поэтому все критические значения бимногочлена-прообраза равны нулю.

$1^{\circ}$. Предположим, что бимногочлен-прообраз точки $u=0$ собственный.

В этом случае $H(Z, \gamma / Z)=h(Z) / Z^{p}$, где $h$ - многочлен степени $n$ (со старшим коэффициентом 1 и свободным членом $\left.\gamma^{p}\right)$. Если $H$ имеет 0 критическим значением кратности $m_{i}$ в критической точке $Z_{i}$, то $h$ имеет в этой точке корень кратности $m_{i}+1$. Поэтому многочлен $h$ должен иметь больше, чем $\sum m_{i}=n$, ненулевых корней (с учетом кратностей), что для многочлена степени $n$ невозможно.

$2^{\circ}$. Предположим, что бимногочлен-прообраз точки $u=0$ несобственный.

В этом случае из леммы вытекает, что

(i) все критические значения многочлена $A$ равны нулю;

(ii) все критические значения многочлена $B$ равны нулю;

(iii) критическое значение $c$ бимногочлена $H$ в точке 0 равно нулю.

Значит, многочлен $A$ имеет вид $\left(W-W_{*}\right)^{p}$. Но свободный член $A$ равен нулю. Следовательно, $W_{*}=0$ и $A=W^{p}$. Точно так же $B=Z^{q}$. Окончательно $H=W^{p}+Z^{q}, \gamma=0$, что и требовалось доказать.

ТЕоремА. Отображение Л3 задает накрытие над пространством многочленов без кратных корней: это пространство накрьввается многообразием бимногочленов без кратных критических значений. Кратность этого накрытия равна числу $N(p, q)$, указанному во введении.

ЗАмЕчАНИЕ. Укороченное отображение задает аналогичное накрытие над дополнением к $(n-2)$-мерному ласточкину хвосту в пространстве $\mathbb{C}^{n-1}$ многочленов с нулевым следом. Кратность его такая же. 
ДоКАЗАТЕЛЬСТВо. Отображение ЛЗ полиномиально и конечнократно по предыдущим теоремам. Кроме того, оно квазиоднородно, с положительными весами аргументов

$$
\operatorname{deg} a_{i}=i q, \quad \operatorname{deg} b_{j}=j p, \quad \operatorname{deg} c=p+q, \quad \operatorname{deg} \gamma=p q
$$

(эти веса диктуются выбором $\operatorname{deg} W=q, \operatorname{deg} Z=p$ ). Веса критических значений при этих весах аргументов равны $\operatorname{deg} H=p q$. Поэтому веса их симметрических функций равны $\operatorname{deg} u_{k}=k p q$.

По общей теореме о квазиоднородных отображениях (см., например, [6]) мы находим кратность отображения Л3 в виде

$$
\begin{aligned}
N(p, q) & =\frac{\prod_{k=1}^{n} \operatorname{deg} u_{k}}{\left(\prod_{i=1}^{p-1} \operatorname{deg} a_{i}\right)\left(\prod_{j=1}^{q-1} \operatorname{deg} b_{j}\right)(\operatorname{deg} c)(\operatorname{deg} \gamma)} \\
& =\frac{\prod_{k=1}^{n}(k p q)}{(p+q) p q \prod_{i=1}^{p-1}(i q) \prod_{j=1}^{q-1}(j p)}=\frac{p^{p} q^{q}(p+q-1) !}{(p-1) !(q-1) !} .
\end{aligned}
$$

Согласно общей теории положительно квазиоднородных конечнократных отображений, отображение Л3 задает накрытие над дополнением к множеству своих критических значений. Найдем множества критических точек и критических значений отображения Л3. Докажем, что критические точки - это бимногочлены с кратными критическими значениями, а критические значения - многочлены, имеющие кратный корень.

ЛЕмма. Якобиан отображения Л3 не обрашается в 0 в точках, где все $n$ критических значений $H_{i}$ различны (и равен нулю в точках, где некоторые из них совпадают, если бимногочлен собственньй).

ДокАЗАТЕЛЬСТВо. Бимногочлен с $n$ различными критическими значениями является собственным. Поэтому мы можем использовать в качестве координаты на гиперболе $Z W=\gamma$ величину $W$ и исследовать критические значения гладкой функции

$F=W^{p}+a_{1} W^{p-1}+\cdots+a_{p-1} W+c+b_{q-1} \gamma W^{-1}+\cdots+b_{1} \gamma^{q-1} W^{1-q}+\gamma^{q} W^{-q}$

от переменной $W$, зависящей от параметров $(a, b, c ; \gamma)$.

Производная критического значения гладкой функции по параметру равна производной самой функции по параметру в критической точке. Поэтому производные критических значений $F_{l}$ в критических точках $W_{l}$ по параметрам $\left(a_{i}, c, b_{j}\right)$ равны $W_{l}^{p-i}, 1$ и $\gamma^{q-j} W_{l}^{j-q}$, а производная по $\gamma$ равна сумме $q \gamma^{q-1} W_{l}^{-q}$ с линейной комбинацией величин $W_{l}^{-1}, \ldots, W_{l}^{1-q}$ с не зависящими от $l$ коэффициентами.

Итак, определитель Якоби критических значений по параметрам $(a, c, b ; \gamma)$ равен произведению величины

$$
\gamma^{1+\cdots+(q-1)} q \gamma^{q-1}\left(\prod W_{l}\right)^{-q}
$$

на определитель Вандермонда $n$ критических точек $W_{l}$

$$
\Delta[W]=\prod_{i>j}\left(W_{i}-W_{j}\right) .
$$


Произведение чисел $W_{l}$ отлично от нуля (дифференцируя $F$ по $W$, мы получаем по формуле Виета $\left.\prod W_{l}=-q \gamma^{q}(-1)^{n} / p\right)$. Окончательно мы получили для определителя Якоби критических значений по параметрам выражение

$$
\left|\partial\left(F_{l}\right) / \partial(a, c, b ; \gamma)\right|=\Delta[W] K \gamma^{-r},
$$

где $r=\left(q^{2}-q+2\right) / 2, K=p^{p} q^{1-q}(-1)^{q(n-1)}, n=p+q$.

Определитель Якоби $n$ первых основных симметрических функций от $F_{l}$ по $F_{l}$ обращается в нуль только на зеркалах, где $F_{i}=F_{j}$.

Действительно, этот определитель легко вычисляется явно и равен

$$
\left|\partial \sigma\left(F_{l}\right) / \partial\left(F_{l}\right)\right|=\Delta\left[F_{i}\right] L, \quad L=(-1)^{n(n-1) / 2} / n .
$$

Таким образом, определитель Якоби отображения Л3 представляет собой (с точностью до множителя, зависящего лишь от $p$ и $q$ ) произведение

$$
\Delta\left[W_{l}\right] \Delta\left[F_{l}\right] \gamma^{-r}
$$

определителей Вандермонда критических точек и критических значений на ненулевой масштабный множитель. Лемма доказана.

Из леммы следует, что полным прообразом многообразия многочленов без кратных корней при отображении Л3 является множество всех бимногочленов без кратных критических значений и что отображение этого множества на многообразие многочленов без кратных корней является накрытием (собственность этого локального диффеоморфизма - общий факт теории положительно квазиоднородных конечнократных отображений).

ЗАмЕЧАНИЕ. Несобственные бимногочлены общего положения (у которых все $n-2$ критических значений на обеих осях различны и отличны от значения в нуле) не являются критическими точками отображения Л3 (хотя и отображаются им в множество его критических значений).

Чтобы в этом убедиться, достаточно оценить расстояние между критическими значениями в обеих критических точках, стремящихся к нулю при $\gamma$, стремящемся к нулю. Из определяющих критические точки формул $Z B_{Z}-W A_{W}=0$, $Z W=\gamma$ находим (при $a_{p-1} b_{q-1} \neq 0$, что мы будем предполагать) первые по $\gamma$ приближения к критическим точкам в виде $W_{ \pm}= \pm u \sqrt{\gamma}+\ldots, u \neq 0$. Для соответствующих критических значений $F_{ \pm}=c \pm v \sqrt{\gamma}+\ldots$ находим их симметрические функции $\sigma_{1}=2 c+\ldots, \sigma_{2}=c^{2}-v^{2} \gamma+\ldots$ Определитель Якоби $\partial\left(\sigma_{1}, \sigma_{2}\right) / \partial(c, \gamma)$ в нуле равен $-\gamma^{2}$, т. е. не равен 0 . Поэтому и полный определитель Якоби отображения Л3 отличен от нуля.

СлЕдСТВИЕ. Многообразие бимногочленов бистепени $(p, q)$ без кратных критических значений является пространством Эйленберга-Маклейна $K(\pi, 1)$, дде $\pi$ - подгруппа индекса $N(p, q)$ в группе кос из $n=p+q$ нитей.

Как уже отмечено выше, все бимногочлены без кратных критических значений собственные.

\section{§4. Топологическая классификация многочленов Лорана}

Теорема. Число $M(p, q)$ попарно топологически неэквивалентных многочленов Лорана с полюсами порлдков $p$ u $q$, имеюших $p+q$ заранее фик- 
сированных попарно различных конечных критических значений, равно

$$
M(p, q)=\frac{N(p, q)}{p q}=\frac{p^{p} q^{q}(p+q-1) !}{p ! q !}
$$

если $p \neq q ; M(p, q)$ вдвое меньше этого значения, если $p=q>1$, и равно 1 при $p=q=1$.

ДокАЗАТЕЛЬСтво. Топологическая эквивалентность двух многочленов Лорана является голоморфным (следовательно, дробно-линейным) отображением сферы-прообраза в сферу-прообраз, переводящим полюс порядка $p$ (соответственно q) первого отображения в полюс такого же порядка второго.

Всякий многочлен Лорана с полюсами порядков $p$ и $q$ топологически эквивалентен собственному бимногочлену бистепени $(p, q)$. Число таких бимногочленов с данными $p+q$ конечными критическими значениями равно $N(p, q)$. Но некоторые из этих бимногочленов топологически эквивалентны. Подсчитаем, сколько среди них топологически не эквивалентных.

$1^{\circ}$. Случай 1. $p \neq q$. Докажем, что число бимногочленов, топологически эквивалентных данному, среди наших $N(p, q)$ бимногочленов равно $p q$.

Топологическая эквивалентность отображений Лорана $z \mapsto f(z)$ с полюсами разных порядков $p \neq q$ в нуле и в бесконечности обязательно линейна (имеет вид $z=\lambda z^{\prime}, \lambda \neq 0$ ). Поэтому соответствующие топологически эквивалентным друг другу многочленам Лорана бимногочлены переводятся друг в друга заменами переменных вида $W=\alpha W^{\prime}, Z=\beta Z^{\prime}$. Чтобы такая замена переводила бимногочлен бистепени $(p, q)$ в бимногочлен, необходимо и достаточно, чтобы $\alpha^{p}=1$ и $\beta^{q}=1$.

ЛЕмма. Бимногочлень, получаюшиесл из данного бимногочлена с $p+q$ различными конечными критическими значениями всеми рq заменами указанного вида, попарно различны.

ДоКАЗАТЕЛЬСТво ЛЕммЫ. Достаточно проверить, что бимногочлен при такой замене не может перейти в себя.

Если бы бимногочлен переходил в себя при такой замене, то мы имели бы $\alpha \beta=1$. Поэтому с ненулевыми коэффициентами в бимногочлен могут входить только такие одночлены $W^{r}$ и $Z^{r}$, для которых $\alpha^{r}=1$. Предположим, что $\alpha=e^{2 \pi i K / L}$, где $K$ и $L$ взаимно просты. Условие $\alpha^{r}=1$ приводит к делимости $K r$ на $L$ и, следовательно, к делимости $r$ на $L$. Поэтому наш бимногочлен можно представить в виде собственного бимногочлена от $W^{L}=\widetilde{W}$ и $Z^{L}=\widetilde{Z}$ (бистепени $(p / L, q / L))$.

Число конечных попарно различных критических значений такого бимногочлена не превосходит $(p+q) / L$, что меньше $p+q$. Поэтому все $p q$ бимногочленов, получаемых из данного нашими заменами, различны. Лемма доказана.

Так как никаких других топологических эквивалентностей между нашими $N$ бимногочленами нет, число классов $M$ равно $N /(p q)$.

$2^{\circ}$. Случай $p=q$. Докажем, что число бимногочленов, топологически эквивалентных данному, среди наших $N(p, q)$ бимногочленов равно $2 p q$ (при $p>1)$. И ответ $M=N /(p q)$, и доказательство сохранились бы и при $p=q$, если бы мы потребовали, чтобы эквивалентность переводила полюс в нуле в полюс в нуле. Поскольку мы, однако, допускаем перестановку полюсов, наша группа замен $(W=$ $\left.\alpha W^{\prime}, Z=\beta Z^{\prime}\right)$ расширяется добавлением перестановки $(Z, W) \mapsto(W, Z)$. 
Лемма. Бимногочлены, получаемые из данного бимногочлена бистепени $(p, p)$ c $2 p$ различными конечными критическими значениями всеми $2 p^{2}$ заменами указанного вида, попарно различнь при $p>1$.

ДокАЗАТЕЛЬСТво ЛЕммы. Достаточно проверить, что наш бимногочлен $H$ не может перейти в себя при подстановке

$$
W=\alpha Z^{\prime}, \quad Z=\beta W^{\prime} .
$$

Если бы такая подстановка переводила $H$ в себя, то было бы $\alpha \beta=1$. Поэтому члены $a W^{r}$ и $b Z^{r}$ в $H$ должны были бы удовлетворять условию $b=a \alpha^{r}$. Рассмотрим еще число $\delta=\sqrt{\alpha}$. Мы получаем $a W^{r}+b Z^{r}=a \delta^{r}\left(\delta^{r} Z^{r}+\delta^{-r} W^{r}\right)$. Выражение в скобках можно записать в виде многочлена от величины $U=$ $\delta Z+\delta^{-1} W$ (степени $r$ ). Мы получаем выражение нашего бимногочлена $H$ в виде многочлена $f$ степени $p$ от одной переменной $U$.

Критические точки $H$ определяются из условия $f^{\prime}(U)(d U / d W)=0$. Поэтому критическими значениями $H$ являются критические значения $f$ и значения $f$ в двух точках, где $d U / d W=0$. Их число $p-1+2$ меньше $2 p$ при $p>1$. Поэтому бимногочлен $H$ с $2 p$ различными конечными критическими значениями не может перейти в себя при нашей подстановке. Лемма доказана. Тем самым и теорема доказана при $p=q>1$.

При $p=q=1$ и доказывать нечего, так как $N=1$ и эквивалентным $H$ быть не с кем.

СлЕдСТВИЕ 1. Многообразие классов топологической эквивалентности многочленов Лорана с полюсами порлдков $p$ и $q$ u c $p+q$ попарно различными критическими значениями естественно накрывается многообразием бимногочленов бистепени $(p, q)$ с $p+q$ попарно различными критическими значениями (с кратностью $p q$, если $p \neq q, 2 p q$, если $p=q>1, u 1$, если $p=q=1)$ и естественно накрывает дополнение к ласточкиному хвосту в пространстве $\mathbb{C}^{n-1}(n=p+q)$ с кратностью $M(p, q)$.

СлЕдСтвИЕ 2. Это многообразие классов многочленов Лорана является пространством Эйленберга-Маклейна $K(\pi, 1)$ для соответствующей подгруппь индекса $M(p, q)$ в группе кос из $n$ нитей.

ДокАЗАТЕльСТво. Конечная группа указанных выше подстановок (порядка $p q$ при $p \neq q$ и $2 p q$ при $p=q>1$ ) действует на многообразии бимногочленов бистепени $(p, q)$ с $p+q$ попарно различными критическими значениями, как мы показали выше, без неподвижных точек. Многообразие орбит этого действия и есть многообразие классов многочленов Лорана, так как каждый такой многочлен допускает запись в виде бимногочлена, причем топологически эквивалентным многочленам Лорана отвечают бимногочлены из одной орбиты.

Этим доказано следствие 1. Следствие 2 прямо вытекает из следствия 1 и результата $\S 3$ о многообразии бимногочленов и его отображении Л3.

СлЕдСТВИЕ 3. Число нумерованных $(n, n)$-графов, перестановка Коксетера которых состоит из двух ииклов длин р и $q$, равно $M(p, q)$.

ДокАЗАТЕльСтво. Согласно последней теореме $\S 2$, это число равно числу листов накрытия, сопоставляющего классу топологической эквивалентности многочлена Лорана с полюсами порядков $p$ и $q$ набор из $p+q$ попарно различных критических значений этого многочлена Лорана. Согласно следствию 1 , это число листов равно $M(p, q)$. 
ЗАМЕЧАНИЕ. Исследование мёбиусовских тригонометрических многочленов (для которых $f(t+\pi)=-f(t))$ сводится к изучению нечетных многочленов Лорана и нечетных бимногочленов. Множество критических значений нечетного бимногочлена четно (инвариантно относительно инволюции изменения знака). Для любого четного множества из $2(p+q+1)$ попарно различных конечных точек сферы-образа существует

$$
\widetilde{N}(p, q)=\frac{(p+q) !}{p ! q !}(2 p+1)^{p+1}(2 q+1)^{q+1}
$$

нечетных бимногочленов с такими критическими значениями и с полюсами порядков $2 p+1$ и $2 q+1$ в 0 и в $\infty$ соответственно. Число $\widetilde{M}$ топологически различных среди них равно

$$
\widetilde{M}(p, q)=\widetilde{N}(p, q) /((2 p+1)(2 q+1)) \text { при } p \neq q,
$$

а при $p=q>0 \widetilde{M}$ вдвое меньше этого выражения $(\widetilde{M}(0,0)=\widetilde{N}(0,0)=1)$.

Соответствующее отображение Л3: $\mathbb{C}^{n} \rightarrow \mathbb{C}^{n}, n=p+q+1$, действует из пространства нечетных бимногочленов в многообразие орбит группы Коксетера $B_{n}$. Пространства нечетных бимногочленов и топологических классов нечетных многочленов Лорана без кратных критических значений накрывают многообразие регулярных орбит группы $B_{n}$ и являются классифицируюшими пространствами $K(\pi, 1)$ для подгрупп индексов $\widetilde{N}$ и $\widetilde{M}$ в группе $\operatorname{Br}\left(B_{n}\right)$ кос Брискорна серии $B$.

Эти результаты приводят к формуле для числа связных нечетно нумерованных графов с $2 k$ ребрами и вершинами.

ОПРЕДЕЛЕНИЕ. Связный граф с $2 k$ вершинами и $2 k$ ребрами, занумерованными числами $( \pm 1, \ldots, \pm k)$ называется нечетно нумерованныл, если на нем действует инволюция, переставляющая ребра с номерами $i$ и $-i$ и не имеющая ни неподвижных вершин, ни неподвижных ребер.

Инволюция такого графа однозначно определяется нумерацией (а с точностью до изоморфизма ненумерованного, но нумеруемого нечетно графа даже и не зависит от нумерации).

ОПРЕДЕЛЕНИЕ. Перестановка Коксетера нечетно нумерованного графа это произведение транспозиций, переставляющих вершины последовательных ребер в порядке $(1, \ldots, k,-1, \ldots,-k)$.

Из наших аналитических построений получается чисто комбинаторное

СлЕдСТвИЕ. Число нечетно нумерованных связных графов с одинаковым числом ребер и вершин, перестановка Коксетера которых состоит из двух ииклов длин $(2 p+1,2 q+1)$, равно $\widetilde{M}(p, q)$.

ПримеР 1. Существует ровно 2 связных графа из четырех ребер и четырех вершин, допускающих нечетную нумерацию:

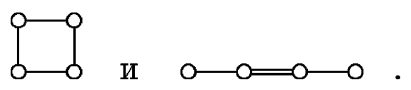

Число нечетных нумераций $\widetilde{M}(0,1)$ равно 3 , так как длины циклов перестановок Коксетера равны 1 и 3. 
ПримеР 2. Существуют ровно 4 нечетно нумеруемых связных графа с 6 вершинами и ребрами. Числа их нечетных нумераций имеют сумму $4+12+12+6=$ 34 . Это число равно сумме чисел $\widetilde{M}(1,1)=9$ и $\widetilde{M}(0,2)=25$, соответствующих разбиениям вершин на циклы $6=3+3$ и $6=1+5$.

ЗАмЕчАниЕ. Длины циклов перестановки Коксетера нечетно нумерованного связного графа с одинаковым числом вершин и ребер нечетны. Это следует из нашей аналитической теории.

\section{ЛитеРАТУРА}

1. Davis C. Extrema of a polynomial. Am. Math. Monthly, 64, 679-680 (1957).

2. Thom $R$. L'equivalence d'une fonction différentiable et d'un polynome. Topology, 3, 297-307 (1965).

3. Здравковска $C$. Топологическая классификация полиномиальных отображений. УМН, 25, вып. 4, 179-180 (1970).

4. Арнольд В. И. Критические точки функций и классификация каустик. УМН, 29, вып. 2, 243-244 (1994).

5. Looijenga $E$. The complement of the bifurcation variety of a simple singularity. Invent. Math., 23, 105-116 (1974).

6. Арнольд В. И., Варченко А. Н., Гусейн-Заде С. М. Особенности дифференцируемых отображений. Т. 1, 2, Наука, М. $(1982,1984)$.

7. Горюнов B. В. Геометрия бифуркационных диаграмм простых проекций на прямую. Функц. анализ и его прил., 15, вып. 2, 77-82 (1981).

8. Goryunov $V . V$. Subprincipal Springer cones and morsifications of Laurent polynomials and $D_{\mu}$ singularities. Adv. Sov. Math., Singularities and Bifurcations (V. I. Arnold ed.), Vol. 21 (1994), pp. 163-188.

9. Арнольд В. И. Топологические инварианты алгебраических функций. II. Функц. анализ и его прил., 4, вып. 2, 1-9 (1970).

10. Натанзон С. М. Униформизация пространств мероморфных функций. ДАН CCCP, 287, № 5 (1986).

11. Kazarian M. E. Singularities of functions on the circle and relative Morse theory. Submitted to Topology. Preprint: Stockholm University (1995).

12. Kazarian M. E. Nonlinear version of Arnold's theorem on flattening points. C. R. Acad. Sci. Paris (1996).

13. Казарян М. Э. Относительная теория Морса одномерных расслоений и циклические гомологии. Функц. анализ и его прил., 30 (1996), в печати.

14. Arnold V. I. Springer numbers and morsification spaces. J. Alg. Geom., 1, 197-214 (1992).

15. Arnold $V . I$. Bernoulli-Euler updown numbers associated with function singularities, their combinatorics and arithmetics. Duke Math. J., 63, № 2, 537-555 (1991).

16. Арнольд В. И. Исчисление змей и комбинаторика чисел Бернулли, Эйлера и Спрингера групп Коксетера и пространств морсификации. УМН, 47, вып. 1, $1-51(1992)$.

17. Арнольд В. И. Топологические проблемы теории распространения волн. УМН, 51, вып. 1, 1-47 (1966).

Математический институт РАН им. Стеклова

и Университет Париж-Дофин

Поступило в редакцию 15 декабря 1995 г. 